\title{
Spheroid body myopathy
}

INSERM

\section{Source}

INSERM. (1999). Orphanet: an online rare disease and orphan drug data base. Spheroid body myopathy. ORPHA:268129

Spheroid body myopathy is a rare form of myofibrillar myopathy characterized by predominantly proximal muscle weakness (that could be either non- or slowly progressive), associated with spheroid body inclusions (composed of myofilamentous material within individual muscle fibers) in skeletal muscle biopsy. Presentation is varied and may range from asymptomatic to severe muscle weakness that manifests with absent Achilles reflexes, gait abnormality and/or other motor incapacitations. 